\title{
Long Wavelength Plasmonic Absorption Enhancement in Silicon Using Optical Lithography Compatible Core-Shell-Type Nanowires
}

\author{
Mohammed Shahriar Sabuktagin, ${ }^{1}$ Khairus Syifa Hamdan, ${ }^{1}$ Khaulah Sulaiman, \\ Rozalina Zakaria, ${ }^{2}$ and Harith Ahmad ${ }^{2}$ \\ ${ }^{1}$ Department of Physics, Faculty of Science, University of Malaya, 50603 Kuala Lumpur, Malaysia \\ ${ }^{2}$ Photonics Research Center, University of Malaya, 50603 Kuala Lumpur, Malaysia
}

Correspondence should be addressed to Mohammed Shahriar Sabuktagin; slhahriar@yahoo.com

Received 24 January 2014; Accepted 19 March 2014; Published 10 April 2014

Academic Editor: Hongtao Yu

Copyright (C) 2014 Mohammed Shahriar Sabuktagin et al. This is an open access article distributed under the Creative Commons Attribution License, which permits unrestricted use, distribution, and reproduction in any medium, provided the original work is properly cited.

\begin{abstract}
Plasmonic properties of rectangular core-shell type nanowires embedded in thin film silicon solar cell structure were characterized using FDTD simulations. Plasmon resonance of these nanowires showed tunability from $\lambda=750 \mathrm{~nm}$ to $\lambda=2400 \mathrm{~nm}$ with variation of dimensional parameters within the feature resolution specifications of Deep Ultraviolet and Laser Interference Lithography techniques. A half-shell nanowire structure was proposed for simplifying device integration which showed 10 times absorption enhancement in silicon at $\lambda=940 \mathrm{~nm}$. However this absorption was significantly smaller than the Ohmic loss in the silver shell due to very low near-bandgap absorption properties of silicon. Prospect of improving enhanced absorption in silicon to Ohmic loss ratio by utilizing dual capability of these nanowires in boosting impurity photovoltaic effect and efficient extraction of the photogenerated carriers was discussed. Our results indicate that high volume fabrication capacity of optical lithography techniques can be utilized for plasmonic absorption enhancement in thin film silicon solar cells over the entire long wavelength range of solar radiation.
\end{abstract}

\section{Introduction}

Thin film solar cells are expected to play a prominent role in clean energy generation. Further improvement in efficiency and reliability is needed to materialize this prospect. Silicon is one of the main materials in thin film solar cell development due to earth abundance and low cost. Complete absorption of solar radiation in thinner films and effective extraction of photogenerated carriers are the key challenges in improving efficiency of thin film silicon solar cells. A number of methods such as light trapping, impurity band photovoltaic effect, and upconversion have been suggested for increasing absorption in next generation silicon solar cells [1-3]. Impurity photovoltaic effect can convert sub-bandgap photons to charge carriers through transitions involving deep level impurities $[1,4]$. Impurity photovoltaic zones can be implemented using a number of conventional doping methods [1]. However, recombination in the impurity zone limits net gain in carrier generation. Light trapping was shown to shift the balance towards increased net carrier generation [4]. Plasmonic effect has drawn strong interest as a light trapping method for enhancing absorption [13]. Plasmonic light trapping utilizes plasmon resonance in metal nanostructures for increasing absorption by scattering or coupling solar radiation into solar cell materials and/or local enhancement of electric field intensities of incident light near the surface of the nanostructures [2]. A variety of nanostructures such as nanoparticles, nanowires, and gratings have been proposed for plasmonic enhancement. These nanostructures are typically less than $100 \mathrm{~nm}$ in size and their practical incorporation in production scale solar cell devices still remains a challenge. Self-organized layers of nanoparticles can be formed by annealing thin silver or gold films [5]. Throughput of this technique is limited by 
long annealing times. Metal nanoparticles and nanowires can be grown by a number of methods [6] and applied to solar cells by spin coating. However spin coating does not allow placement of the nanoparticles with controlled spacing. Aggregation of the nanoparticles causes large change of their plasmonic properties due to coupling, and sparse distribution makes plasmonic enhancement less than optimal. Ebeam lithography, Nano Imprint Lithography (NIL), and Substrate Conformal Imprint Lithography (SCIL) can be used for forming regular arrays of sub-100 $\mathrm{nm}$ structures [7]. These techniques are yet to meet the low cost large-scale fabrication requirements of solar cell technologies. Ebeam lithography can be applied to small areas, and throughput is low due to sequential nature of the process. Both NIL and SCIL need highly clean environment. NIL has stringent surface flatness and roughness requirements. SCIL technique has been developed to relax the surface quality requirements of NIL so that it can become the volume production technique for nanofabrication. Still SCIL needs high resolution ebeam lithography for making master masks and special equipment for replicating production masks which have limited lifetime. On the other hand, Deep Ultra Violet Lithography (DUVL) and Laser Interference Lithography (LIL) techniques can be used for large area high volume fabrication of photonic nanostructures with feature sizes larger than $100 \mathrm{~nm}[8,9]$. LIL is particularly suitable for making periodic grating type patterns in square meter scale areas without requiring a mask [9]. In this work, FDTD simulations were performed to explore the feasibility of using DUVL and LIL fabricated nanostructures for plasmonic absorption enhancement in silicon solar cells. Core-shell type rectangular nanowires were chosen in our studies since plasmon resonance of core-shell type structures can be tuned widely by varying core or shell dimensions [10, 11], and rectangular shape is compatible with planar thin film fabrication technologies. Light trapping properties of core-shell type structures were discussed in [12, 13]. Analytical solutions for spherical core-shell particles were reported in [14]. Our simulation results show that plasmon resonance wavelength of these nanostructures can be tuned from $\lambda=750 \mathrm{~nm}$ to $\lambda=2400 \mathrm{~nm}$ by adjusting dimensional parameters. These plasmonic structures may improve the performance of impurity photovoltaic solar cells by serving the dual purpose of absorption enhancement and effective separation photogenerated carriers.

\section{Structure of the Nanowires and Suggested Fabrication Process}

Figure 1 shows a core-shell nanowire in the simulation set-up discussed next. The dimensional parameters of the nanowire such as width, height, and shell thickness are also shown in this figure. Figure 2 shows suggested fabrication sequence and structure (Figure 2(d)) of a half-shell nanowire. Photolithography (Figure 2(a)) and etching (Figure 2(b)) can be used for defining the width and height of the nanowires. A metallization method with good step coverage such as sputtering or ebeam evaporation with sample rotation should

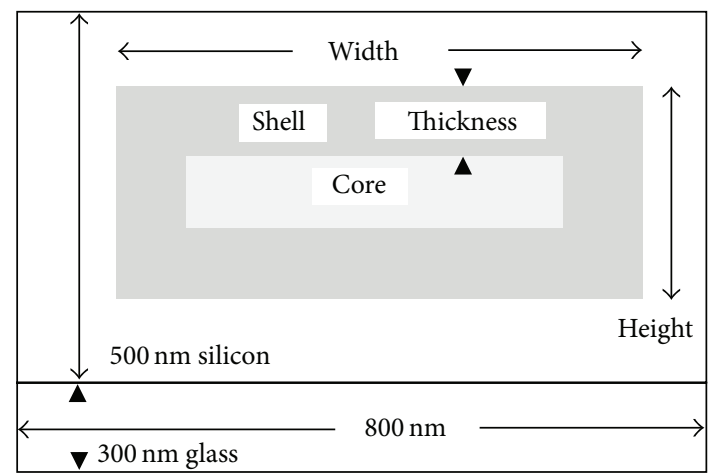

FIGURE 1: Sketch of a core-shell nanowire in the simulation set-up.

be used for depositing the shell layer (Figure 2(c)). For halfshell nanowire fabrication, the next step is lift-off followed by silicon deposition to embed the nanowires in silicon. For core-shell nanowires, silicon deposition should be done next for forming the core. A directional deposition method such as ebeam evaporation without sample rotation should be used for silicon deposition so that metal on the sidewalls (Figure 2(c)) does not get coated with silicon. Residual silicon from the sidewalls can be removed by mild etching. Then deposition of the top metal layer and lift-off would complete the core-shell nanowire. These fabrication steps and structures are compatible with polycrystalline silicon solar cell development using ebeam evaporation [15].

\section{Simulation Set-Up}

Simulations were performed in 2D using Lumerical FDTD Solutions [16]. Nanowires significantly longer than the wavelength of incident light can be considered infinitely long in the context of light scattering. The nanowires fabricated by DUVL or LIL techniques can be much longer than the optical wavelengths in solar radiation. Hence these nanowires can be assumed to be infinitely long, and cross-sectional 2D simulation can be applied to study their localized plasmon properties. Silver and silicon models were based on data from Palik [17]. Staircase approximation was applied at the material boundaries. Mesh size was at least 10 times smaller than the smallest feature present in the simulation. The nanowires were positioned at the center of an $800 \times$ $800 \mathrm{~nm}$ simulation cell with Perfectly Matched Layer (PML) boundary. $300 \mathrm{~nm}$ of glass substrate and $500 \mathrm{~nm}$ of silicon layer were included in the simulation cell (Figure 1). Same parameters of the Total Field Scattered Field (TFSF) source were used in all related simulations so that power absorption data from different simulation runs could be compared. Spatial distribution of optical power absorption was recorded using Lumerical's built-in advanced power monitor for better accuracy. According to Lumerical documentation, electric field component values are interpolated to the center of Yee cells for power absorption calculations. Advanced algorithms are used for more accurate estimation of electric field values at metal-nonmetal boundaries. Power absorption was recorded in a $200 \times 150 \mathrm{~nm}$ box shaped region with the nanowire at 


\begin{tabular}{|c|c|c|}
\hline Photoresist & & Photoresist \\
\hline \multicolumn{3}{|l|}{ Silicon } \\
\hline & Glass & \\
\hline
\end{tabular}

(a)

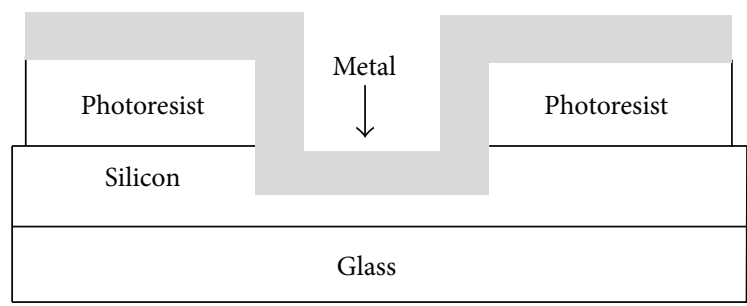

(c)

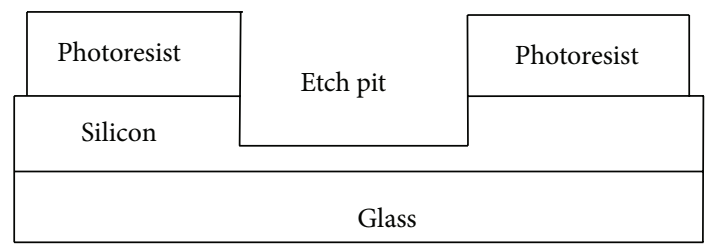

(b)

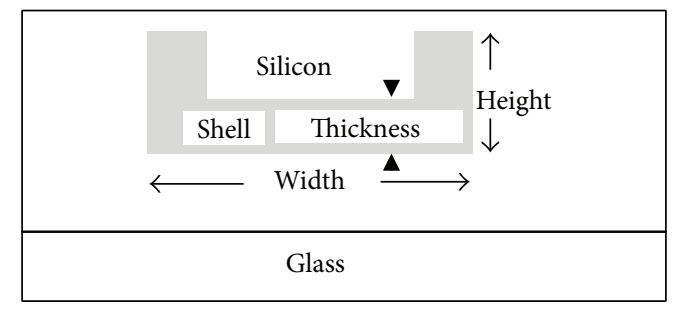

(d)

FiguRE 2: Suggested fabrication sequence for half-shell nanowires. (a) Lithography, (b) etching, (c) metal deposition, and (d) a half-shell nanowire after lift-off and silicon deposition.

the center for all simulations with nanowire widths smaller than $150 \mathrm{~nm}$. Size of the power absorption recording box was $300 \times 150 \mathrm{~nm}$ for wider nanowires. In this geometry, power absorption due to enhanced local electric fields of the nanowire can be assumed to be much larger than absorption due to scattered and incident fields. Plasmon resonance causes an increase in electric field intensities in the metal of the nanowire and in the semiconductor near the nanowire surface. Power absorption at a wavelength " $\omega$ " in a Yee cell with refractive index " $n(x, y, \omega)$ " and electric field intensity " $E(x, y, \omega)$ " is calculated using the following formula:

$$
P_{\text {abs }}(\omega)=0.5 * \omega *|E(x, y, \omega)|^{2} * \operatorname{imag}(n(x, y, \omega)) \text {. }
$$

Summation of absorption from all Yee cells in the simulation area gives the total absorption at a particular wavelength. This process is repeated for all wavelengths. Hence plasmon resonance gives rise to peaks in the power absorption versus wavelength plots. A computer program was used for determining whether a Yee cell is located in silicon or silver. Thus power absorption in silicon and silver regions was determined separately.

\section{Plasmonic Enhancement Properties of Core-Shell and Half-Shell Nanowires}

Figure 3 shows absorption in silicon and silver versus wavelength plots for the nanowires. Core material for the coreshell nanowire was silicon and shell material was silver for both of the nanowires. Keeping the shell thickness constant at $10 \mathrm{~nm}$, the widths and heights of the nanowires were adjusted to bring their plasmon resonance close to $\lambda=950 \mathrm{~nm}$ for simplifying absorption comparison since plasmon resonance wavelength, enhanced electric field distribution and absorption vary with wavelength dependent refractive indices of the metal and surrounding semiconductor. Increase of width and height dimensions caused plasmon resonance to shift to longer wavelengths. Such shift was observed in spherical

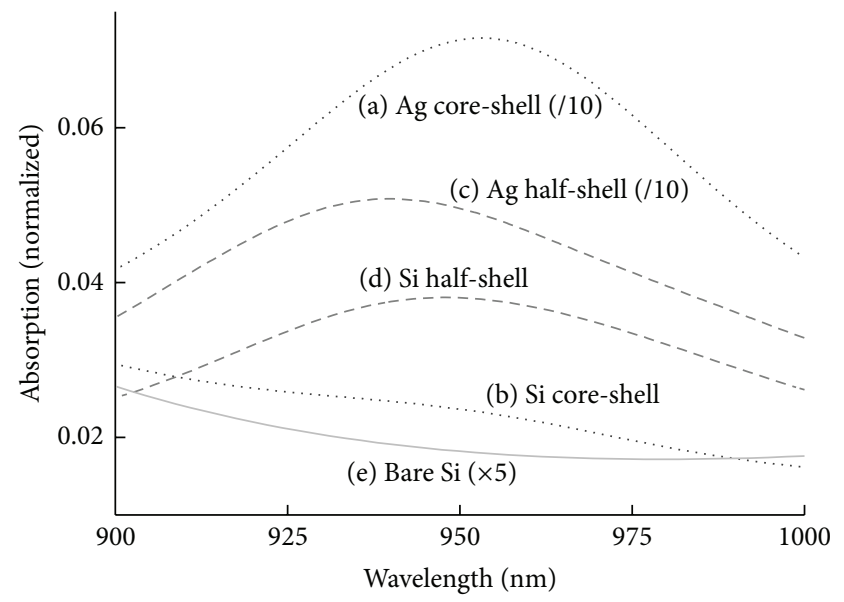

FIgURe 3: Power absorption spectra in silver (traces (a) and (c)) and silicon (traces (b) and (d)) for the core-shell and half-shell nanowires. Absorption in bare silicon without nanowire is shown in (trace (e)). This trace was scaled up by 5 times, and absorption in silver was downscaled by 10 times. Power absorption was normalized with respect to source power.

core-shell nanoparticles for increasing core diameters $[10$, 11]. Plasmon resonance was at $\lambda=951 \mathrm{~nm}$ for a coreshell nanowire with $120 \mathrm{~nm}$ width and $40 \mathrm{~nm}$ height. A halfshell nanowire with $120 \mathrm{~nm}$ width and $35 \mathrm{~nm}$ height showed plasmon resonance at $\lambda=946 \mathrm{~nm}$. Traces (a) and (c) show absorption in silicon for the core-shell and half-shell nanowires. Traces (b) and (d) show absorption in silver downscaled by 10 times for the same nanowires. Trace (e) shows absorption in bare silicon without any nanowire scaled up by a factor 5 for comparison. These traces were scaled for improving graphical representation. Comparing the traces (a), (c), and (e), absorption enhancements in silicon for the core-shell and half-shell nanowires were 7 and 10 times, respectively. 5 times absorption enhancement was reported 


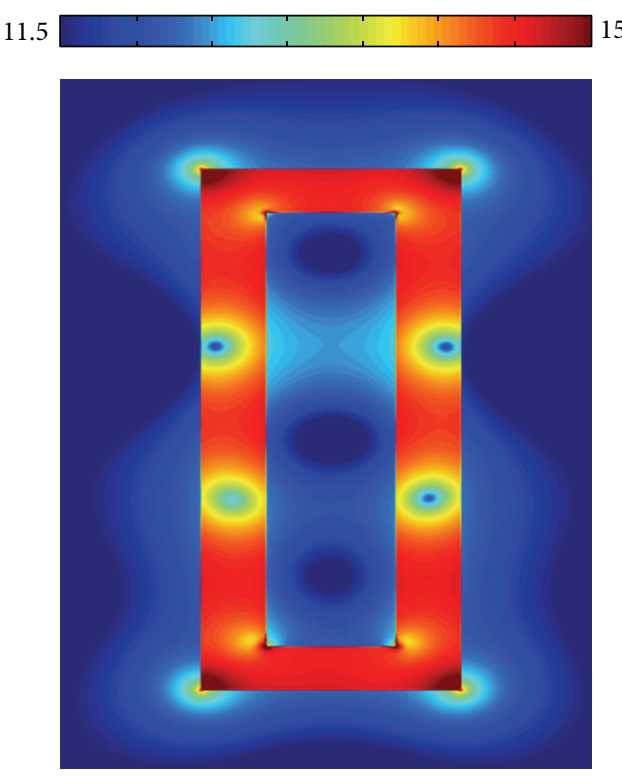

(a)

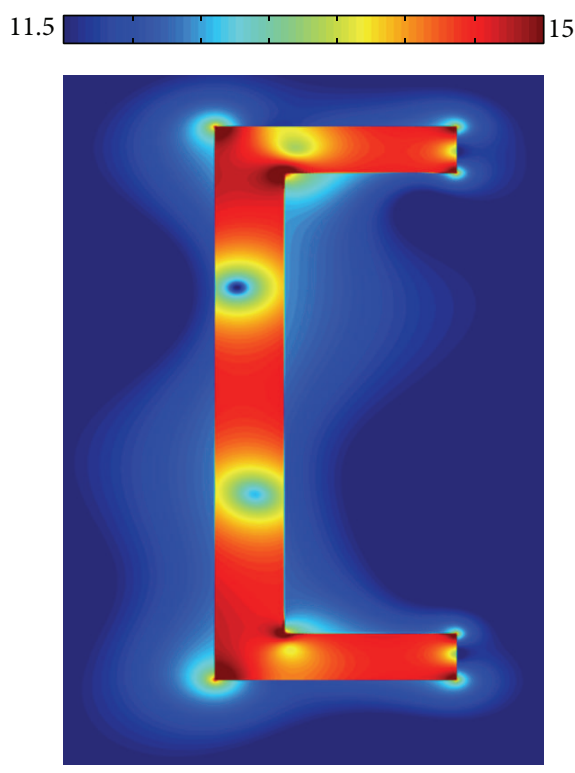

(b)

FIGURE 4: Spatial map of power absorption in logarithmic scale for a (a) core-shell nanowire $(120 \times 40)$ at $\lambda=951 \mathrm{~nm}$. (b) half-shell nanowire $(120 \times 35)$ at $\lambda=946 \mathrm{~nm}$. Shell thickness was $10 \mathrm{~nm}$.

in [18] due to a solid spherical silver nanoparticle at $\lambda=$ $870 \mathrm{~nm}$. Hence geometry of plasmonic nanostructures plays an important role in determining absorption enhancement. Figure 4(a) shows a spatial map of optical power absorption in the vicinity a core-shell nanowire in logarithmic scale. Power absorption was calculated using (1). This figure shows strong absorption in the core region. In commonly used solar cell structures, such as $\mathrm{p}-\mathrm{i}-\mathrm{n}$ or $\mathrm{p}-\mathrm{n}$ type devices, effective extraction of the photogenerated carriers from the core region would require additional device design and fabrication steps. Hence the half-shell nanowire structure was investigated. This nanowire would resemble U-shape for larger nanowire heights. Various truncated structures such as split ring type metamaterials are known to show interesting plasmonic properties which motivated us to investigate this structure. Fortuitously, plasmon resonance of the half-shell nanowires was also tunable in the desired wavelength ranges and their absorption enhancement properties were better than the core-shell nanowires. Spatial distribution of optical power absorption for a half-shell nanowire is shown in Figure 4(b). From this figure, it is apparent that photogenerated carriers can drift towards the collection contacts in conventional solar cell structures without encountering obstacle.

Comparison of the traces (c) and (d) in Figure 3 shows that even the enhanced absorption in silicon was less than $10 \%$ of the Ohmic absorption loss in the silver shell of the halfshell nanowires. Ohmic absorption loss for solid spherical silver nanoparticles was also found to be large which prompted the authors in [14] to hold a pessimistic view regarding effective plasmonic absorption enhancement in silicon. We argue that our proposed plasmonic enhancement structures can potentially lead to more favorable enhanced absorption to Ohmic loss ratio by serving the dual purpose of plasmonic enhancement of impurity optical absorption in silicon and extraction of one type of the photogenerated carriers. Since spherical silver nanoparticles must be isolated from each other in a solar cell structure to preserve their individual plasmonic properties, they cannot play an effective role in carrier transport and extraction. According to the material models used in our simulations, imaginary component of refractive index of silver was 6200 times larger than silicon at $\lambda=940 \mathrm{~nm}$. However Ohmic loss in silver was not 6200 times larger. Hence the spatial distribution of plasmonic electric fields is very effective in enhancing absorption in silicon. Formation of narrow impurity photovoltaic zones around the nanowires may lead to better utilization of such field distribution for enhancing absorption. Impurity photovoltaic effect can be described as electron-hole pair generation by subbandgap photons through transitions involving deep level impurities. Deep level impurities also increase carrier recombination in the impurity zone which hinders the effective separation of generated carriers specially for wider impurity zones. Our proposed structure can resolve this dilemma by serving the dual purpose of plasmonic enhancement and carrier collection. The silver shell can form Ohmic contact to $\mathrm{n}$-type silicon and extract electrons effectively. Reduction of the impurity zone width and immediate collection of one type of the photogenerated carriers would decrease recombination loss in the impurity zone. Since the real parts of refractive index of amorphous silicon $(\mathrm{a}-\mathrm{Si}: \mathrm{H})$ and crystalline silicon are comparable, we speculate that nanowires with similar dimensions embedded in a-Si: $\mathrm{H}$ would show plasmon resonance at nearly the same wavelengths. The poorly absorbing photons on the upper side of crystalline silicon bandgap are sub-bandgap photons for a-Si : $\mathrm{H}$ since the bandgap of a-Si : $\mathrm{H}$ 


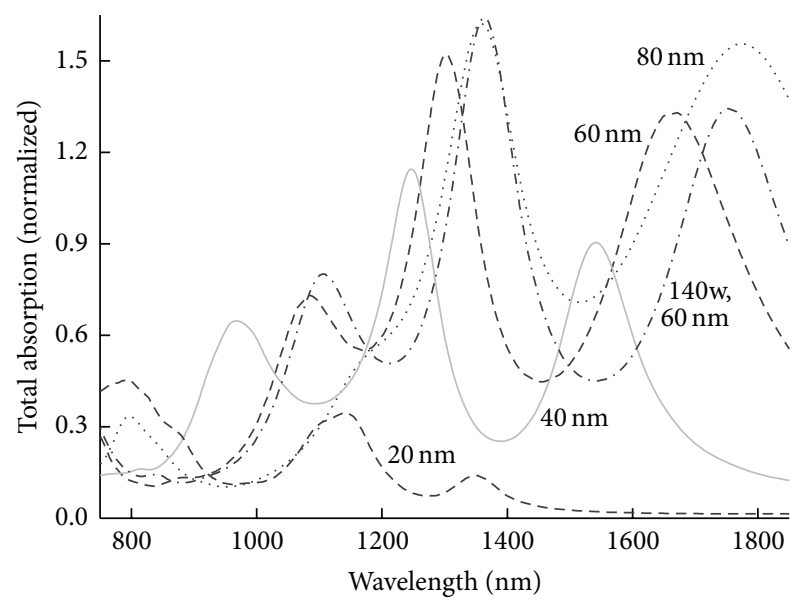

FIGURE 5: Total (silicon + silver) power absorption spectra for halfshell nanowires with heights from 20 to $80 \mathrm{~nm}$. Width of these nanowires was $120 \mathrm{~nm}$. The trace marked as $(140 \mathrm{w}, 60 \mathrm{~nm})$ shows the spectrum for a $(140 \times 60)$ nanowire. Power absorption was normalized with respect to source power.

is larger. Hence these nanowires may be used for enhancing impurity photovoltaic effect in a-Si:H solar cells as well. Plasmonic enhancement of impurity photovoltaic effect in a$\mathrm{Si}: \mathrm{H}$ has been reported recently [19].

\section{Plasmon Resonance Tuning of Half-Shell Nanowires}

Solar radiation has significant power density up to approximately $\lambda=2500 \mathrm{~nm}[20]$ which can be harvested by impurity photovoltaic effect discussed above. Hence tunability of plasmon resonance of the half-shell nanowires was investigated for absorption enhancement at the longer wavelengths of solar spectra. Keeping the shell thickness constant at $10 \mathrm{~nm}$, widths and heights of the nanowires were varied to study their effect on plasmon resonance. Optical power absorption spectra were recorded for the bandwidth $\lambda=750 \mathrm{~nm}$ to $\lambda=1850 \mathrm{~nm}$. Figure 5 shows total power (silicon + silver) absorption spectra for nanowire heights from 20 to $80 \mathrm{~nm}$. Width of these nanowires was $120 \mathrm{~nm}$. Absorption spectrum for a nanowire with $140 \mathrm{~nm}$ width and $60 \mathrm{~nm}$ height is also shown in this figure to demonstrate the effect of width variation. Most of the absorption takes place in silver at longer wavelengths. Absorptions in silicon and silver were not separated since the purpose of this plot is to show the tunability of plasmon resonance. Figure 5 shows three plasmon resonance peaks for each nanowire.

These peaks shift to longer wavelengths with increase of height. It is possible to tune plasmon resonance from $\lambda=750 \mathrm{~nm}$ to $\lambda=1850 \mathrm{~nm}$ by choosing an appropriate nanowire height from $20 \mathrm{~nm}$ to $80 \mathrm{~nm}$ while keeping the nanowire width fixed at $120 \mathrm{~nm}$. Increase of nanowire width also shifts plasmon resonance to longer wavelengths as can be seen by comparing the absorption traces for the 120 $\times 60$ and $140 \times 60 \mathrm{~nm}$ nanowires. The longest plasmon resonance peak for a $250 \times 60 \mathrm{~nm}$ nanowire was located at $\lambda=2400 \mathrm{~nm}$. This nanowire is not shown in Figure 5 since the simulation bandwidth and power absorption recording box size for this nanowire were different from the other nanowires. Simulation bandwidth was from $\lambda=1000 \mathrm{~nm}$ to $\lambda=2500 \mathrm{~nm}$ for this nanowire due to validity limit of silver material model from $\lambda=1 \mu \mathrm{m}$ to $10 \mu \mathrm{m}$. Silver model used in the previous simulations was valid up to $\lambda=2 \mu \mathrm{m}$. Hence plasmon resonance of half-shell nanowires can be tuned in the $\lambda=750 \mathrm{~nm}$ to $\lambda=2400 \mathrm{~nm}$ range by adjusting the width and height dimensions. These nanowires may enhance impurity photovoltaic effect over the entire long wavelength range of solar radiation.

\section{Conclusion}

Numerical investigations on the feasibility of fabricating plasmonic absorption enhancement structures for silicon thin film solar cells using optical lithography techniques were performed. Simulation results indicate that reliable mass production capability of optical lithography techniques can be utilized for fabricating nanowires with plasmon resonance tunability from $\lambda=750 \mathrm{~nm}$ to $\lambda=2400 \mathrm{~nm}$ covering the entire long wavelength range of solar radiation. These nanowires may lead to improved absorption enhancement to Ohmic loss ratio by serving the dual purpose of plasmonic enhancement of impurity photovoltaic effect and effective extraction of photogenerated carriers.

\section{Conflict of Interests}

The authors declare that there is no conflict of interests regarding the publication of this paper.

\section{Acknowledgments}

Dr. Rozalina Zakaria would like to thank the University of Malaya for the Project Grant no. RP008E-13AET. Dr. Sabuktagin and Dr. Ahmad would like to acknowledge Grant No. UM.C/625/1/HIR/MOHE/SCI/29.

\section{References}

[1] A. B. C. López, A. M. Vega, and A. L. López, Next Generation of Photovoltaics, vol. 165 of Springer Series in Optical Sciences, Springer, 2012.

[2] H. A. Atwater and A. Polman, "Plasmonics for improved photovoltaic devices," Nature Materials, vol. 9, no. 3, pp. 205213, 2010

[3] S. Mokkapati and K. R. Catchpole, "Nanophotonic light trapping in solar cells," Journal of Applied Physics, vol. 112, Article ID 101101, 2012.

[4] M. J. Keevers and M. A. Green, "Efficiency improvements of silicon solar cells by the impurity photovoltaic effect," Journal of Applied Physics, vol. 75, no. 8, pp. 4022-4033, 1994.

[5] H. R. Stuart and D. G. Hall, "Absorption enhancement in silicon-on-insulator waveguides using metal island films," Applied Physics Letters, vol. 69, no. 16, pp. 2327-2329, 1996.

[6] D. L. Fedlheim and C. A. Foss, Metal Nanoparticles: Synthesis, Characterization, and Applications, Taylor \& Francis, 2001. 
[7] M. A. Verschuuren, Substrate conformal imprint lithography for nanophotonic [Ph.D. thesis], Utrecht University, 2010.

[8] W. Bogaerts, V. Wiaux, D. Taillaert et al., "Fabrication of photonic crystals in silicon-on-insulator using 248-nm deep UV lithography," IEEE Journal on Selected Topics in Quantum Electronics, vol. 8, no. 4, pp. 928-934, 2002.

[9] B. Bläsi, H. Hauser, O. Höhn, V. Kübler, M. Peters, and A. J. Wolf, "Photon management structures originated by interference lithography," Energy Procedia, vol. 8, pp. 712-718, 2011.

[10] S. J. Oldenburg, J. B. Jackson, S. L. Westcott, and N. J. Halas, "Infrared extinction properties of gold nanoshells," Applied Physics Letters, vol. 75, no. 19, pp. 2897-2899, 1999.

[11] S. J. Oldenburg, R. D. Averitt, S. L. Westcott, and N. J. Halas, "Nanoengineering of optical resonances," Chemical Physics Letters, vol. 288, no. 2-4, pp. 243-247, 1998.

[12] S. K. Kim, R. W. Day, J. F. Cahoon et al., “Tuning light absorption in core/shell silicon nanowire photovoltaic devices through morphological design," Nano Letters, vol. 12, no. 9, pp. 49714976, 2012.

[13] O. Guilatt, B. Apter, and U. Efron, "Light absorption enhancement in thin silicon film by embedded metallic nanoshells," Optics Letters, vol. 35, no. 8, pp. 1139-1141, 2010.

[14] A. L. Aden and M. Kerker, "Scattering of electromagnetic waves from two concentric spheres," Journal of Applied Physics, vol. 22, no. 10, pp. 1242-1246, 1951.

[15] O. Kunz, Z. Ouyang, S. Varlamov, and A. G. Aberle, “ $5 \%$ efficient evaporated solidphase crystallised poly crystalline silicon thin-film solar cells," Progress in Photovoltaics: Research and Applications, vol. 17, no. 8, pp. 567-573, 2009.

[16] "FDTD solutions," https://www.lumerical.com.

[17] E. D. Palik, Handbook of Optical Constants of Solids, Academic Press, 1985.

[18] P. Spinelli and A. Polman, "Prospects of near-field plasmonic absorption enhancement in semiconductor materials using embedded Ag nanoparticles," Optics Express, vol. 20, pp. A641A654, 2012.

[19] F. Lükermann, U. Heinzmann, and H. Stiebig, "Plasmon enhanced resonant defect absorption in thin a-Si:H n-i-p devices," Applied Physics Letters, vol. 100, Article ID 253907, 2012.

[20] http://rredc.nrel.gov/solar/spectra/am1.5/. 

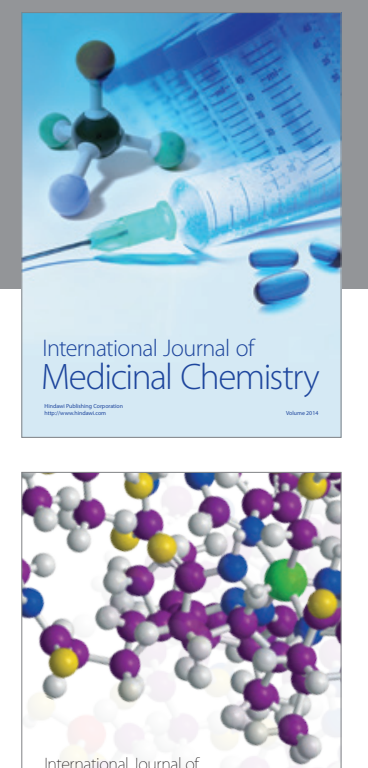

\section{Carbohydrate} Chemistry

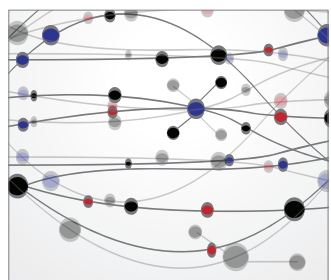

The Scientific World Journal
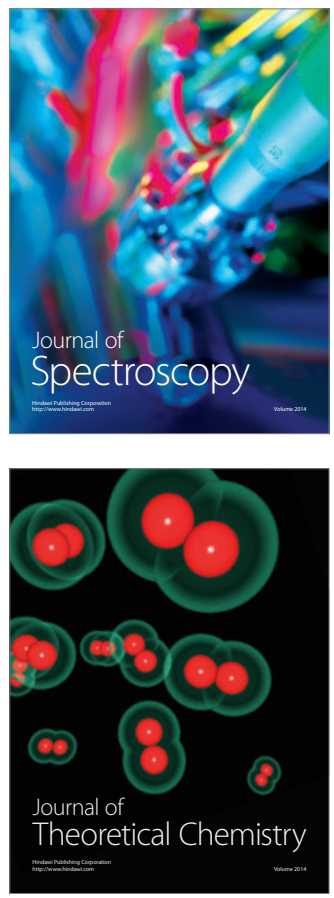
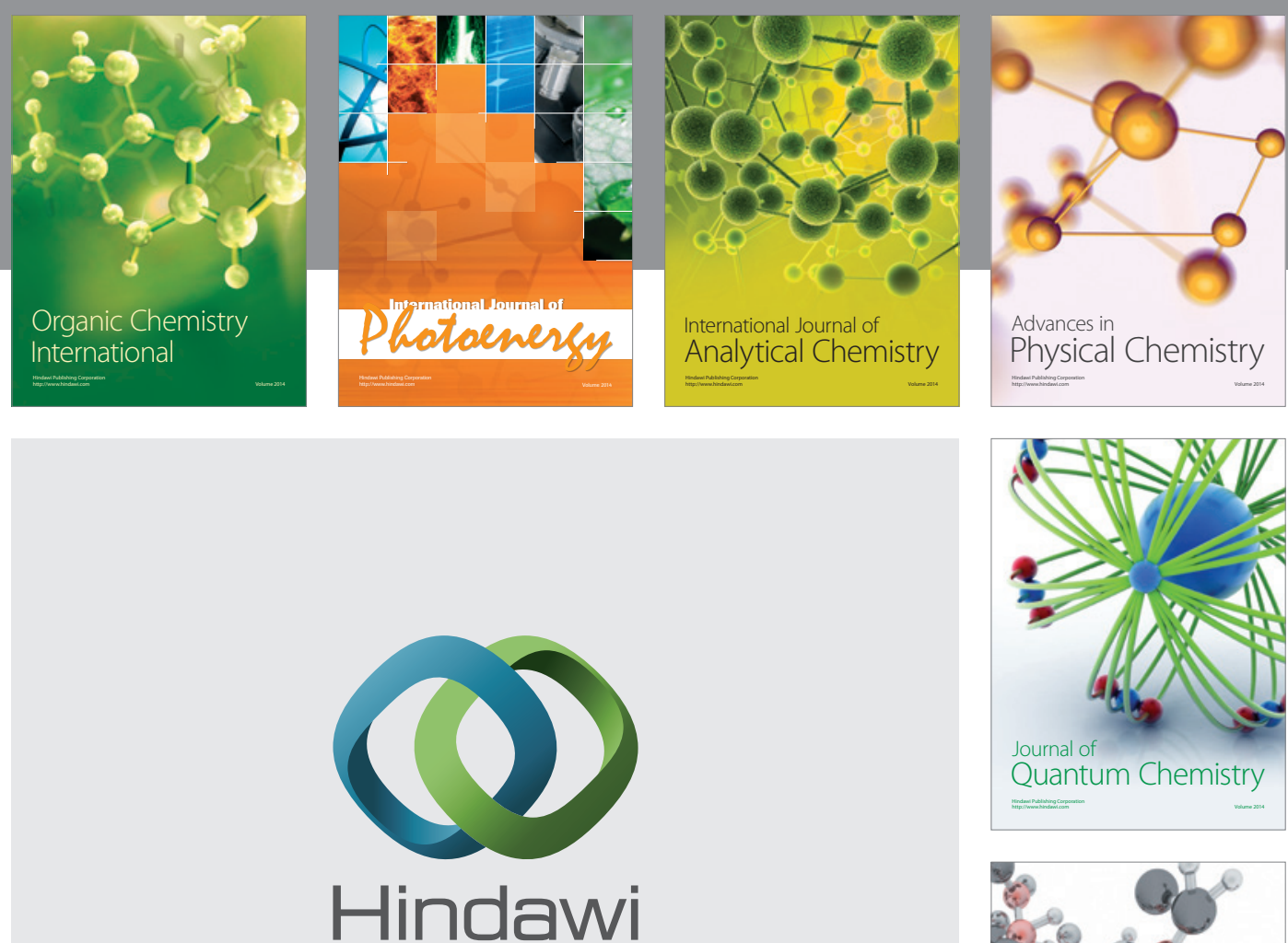

Submit your manuscripts at

http://www.hindawi.com

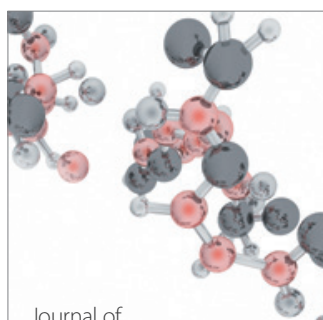

Analytical Methods

in Chemistry

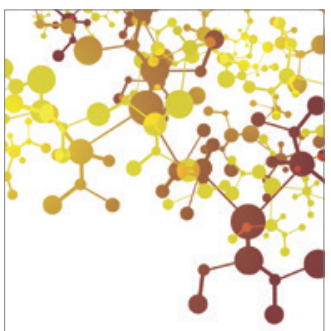

Journal of

Applied Chemistry

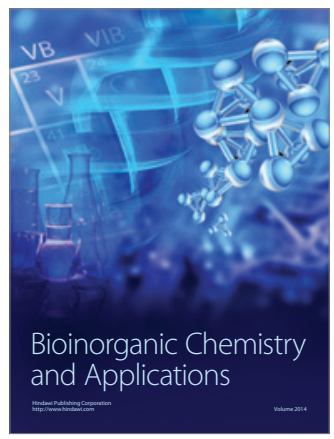

Inorganic Chemistry
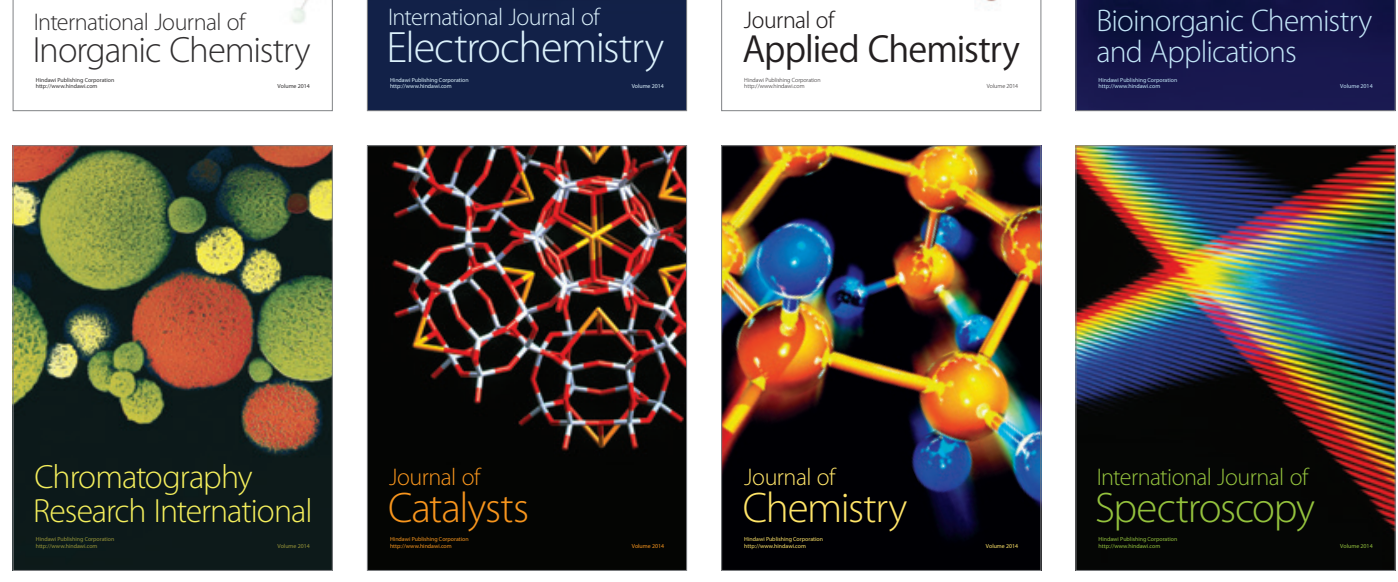